This item was submitted to Loughborough's Research Repository by the author.

Items in Figshare are protected by copyright, with all rights reserved, unless otherwise indicated.

\title{
Experimental study of sound radiation by plates containing circular indentations of power-law profile
}

PLEASE CITE THE PUBLISHED VERSION

http://dx.doi.org/10.1016/j.apacoust.2014.07.014

\section{PUBLISHER}

(C) Elsevier

\section{VERSION}

AM (Accepted Manuscript)

\section{PUBLISHER STATEMENT}

This work is made available according to the conditions of the Creative Commons Attribution-NonCommercialNoDerivatives 4.0 International (CC BY-NC-ND 4.0) licence. Full details of this licence are available at: https://creativecommons.org/licenses/by-nc-nd/4.0/

\section{LICENCE}

CC BY-NC-ND 4.0

\section{REPOSITORY RECORD}

Bowyer, E.P., and Victor V. Krylov. 2014. "Experimental Study of Sound Radiation by Plates Containing Circular Indentations of Power-law Profile". figshare. https://hdl.handle.net/2134/16175. 


\title{
Experimental study of sound radiation by plates containing circular indentations of power-law profile
}

\author{
E.P. Bowyer, V.V. Krylov*
}

Department of Aeronautical and Automotive Engineering, Loughborough University, Loughborough, Leicestershire, LE11 3TU, UK

\begin{abstract}
In this paper, the results of the first experimental investigation into sound radiation of rectangular plates containing tapered indentations of power-law profile are reported. Such tapered indentations materialise two-dimensional acoustic black holes for flexural waves in plates that result in absorption of a large proportion of the incident wave energy. A multiindentation plate was compared to a plain reference plate of the same dimensions, and the radiated sound power was determined according to ISO 3744. It was demonstrated that not only do such multiple indentations provide substantial reduction in the damping of flexural vibrations within the plates, but also cause a substantial reduction in the radiated sound power. As the amplitudes of the flexural vibrations of a plate are directly linked to the
\end{abstract}

\footnotetext{
*Corresponding author. Tel.: +44 1509 227216; E-mail address: V.V.Krylov@lboro.ac.uk
} 
amplitudes of radiated sound from the same plate, this paper also considers the effect of redistribution of the amplitude of the plate's response due to the presence of acoustic black holes on the amplitudes of the radiated sound. The results show that, in spite of some increase in the amplitudes of the displacements at the centres of black holes (circular indentations), the overall reduction of vibration response over the plate is large enough to cause a substantial reduction in the resulting sound radiation from plates containing indentations of power-law profile.

Keywords: Vibration; Rectangular plates; Circular indentations of power-law profile; Acoustic black holes; Vibration damping; Sound radiation.

\section{Introduction}

Demands for noise reduction, especially within the transport industry, are continuously increasing, which stimulates researchers to explore new and more efficient ways of noise suppression at source. Among the variety of different mechanisms of noise generation, the radiation of sound as a result of structural vibration, i.e. 'structure-borne sound', is probably one of the most practically important mechanisms. This paper deals with sound radiation by flexural vibrations of rectangular plates that are designed specifically to reduce the structureborne sound via certain modifications to the geometry of the plates. 
As it is well known, the amplitudes of flexural vibrations of a plate are directly linked to the amplitude of sound radiated from the same plate [1-4], which means that the required reduction in the level of radiated sound can be obtained via efficient damping of structural vibrations of the plate. Passive damping of structural vibrations is traditionally achieved by adding layers of highly absorbing materials to the structure in order to increase energy dissipation of propagating (mostly flexural) waves [5-7]. Another well-known approach to suppression of resonant vibrations of different structures is to reduce reflections of structural waves from their free edges [5, 8].

Over the last decade, a new method of damping flexural vibrations using the so-called 'acoustic black hole effect' to efficiently reduce edge reflections has been developed and investigated [9-11]. This method has been applied to plates containing one- and twodimensional recesses of power-law profile (wedges and circular indentations respectively) that had to be covered by narrow strips of absorbing layers near sharp edges.

In the case of one-dimensional geometry, the equation describing a power-law profile is given by $h(x)=\varepsilon \cdot x^{m}$, where $h(x)$ is the local thickness of the plate, and $\varepsilon$ and $m$ are positive constants. Ideally, if the power-law exponent, $m$ is equal or larger than two, the flexural wave never reaches the sharp edge and therefore never reflects back either [12]. However, this never happens in reality because real wedges always have edge truncations that do not permit the reflection coefficients to be reduced substantially. The addition of a thin strip of absorbing material to the edge of a wedge of power-law profile [9-11] changes this situation dramatically and reduces the reflection coefficient down to $1-3 \%$, which constitutes the 'acoustic black hole effect'. It has been established theoretically [9, 10] and confirmed experimentally [11] that this method of damping structural vibrations is very efficient even in the presence of noticeable edge truncations. This has been demonstrated also by numerical 
calculations combined with experimental testing for rectangular plates with attached powerlaw-shaped wedges [13].

Two-dimensional acoustic black holes, i.e. circular indentations of power law profile, have been considered as well both theoretically and experimentally [14-17]. The practical advantage of two-dimensional acoustic black holes (indentations) over one-dimensional ones (wedges) is that they are placed inside plates. Therefore, they do not have exposed sharp edges that represent health hazard and make it difficult to use the plates with sharp edges as parts of machines and constructions. It has been demonstrated that two-dimensional acoustic black holes are also very efficient for damping structural vibrations.

Note that traditional methods of vibration damping in plate-like structures involve the use of significant quantities of viscoelastic absorbing material on plate surfaces, which is not very efficient and adds significant mass to the plates. The key advantage of the acoustic black hole effect is a significant reduction in added mass of the absorbing material required, as only the edge of a tapered wedge or the centre of a circular power-law profiled indentation have small pieces of absorbing material applied.

In the light of the above-mentioned advantages of acoustic black holes, it is important to consider sound radiation from structures containing such objects. Acoustic black holes damp flexural vibrations in plates over a broad frequency range, and therefore one would expect that sound radiation of the structure should also be reduced. However, it has also been shown that the amplitude of displacement at the wedge or indentation tip of power-law profile is noticeably increased. Therefore, it is essential to consider the implications of such increase in the amplitudes of the displacements at the indentation tips on the sound radiation. In particular, whether the overall reduction in the displacement amplitudes over the constant 
thickness section of the plate is large enough to result in substantial reductions in the overall vibration response and therefore in the resulting sound radiation from plates.

The present paper describes the results of the first experimental investigation of sound radiation from vibrating plates containing indentations of power-law profile. The results for the levels of sound radiation are expressed in terms of radiated sound power. Measurements are carried out for a rectangular steel plate containing multiple indentations of power-law profile, and the results are compared with the results for a plain reference plate of the same size. The results for radiated sound power are also considered in association with visual representations of the vibration displacements over the samples obtained using a scanning laser vibrometer. This helps to understand the observed sound reduction in the light of the amplitude variations of normal displacements over the plates. Part of the material described in this paper has been presented at the 164th ASA meeting (Kansas City, October 2012) and published in POMA [18].

\section{Experimental set up and procedure}

\subsection{Experimental samples and their manufacturing}

Two types of samples were used for this investigation: a plain reference plate and a plate containing six circular indentations of power-law profile (with $m=4$ ), see Fig. 1 . A CNC (Computer Numerically Controlled) milling machine operating at a cutter speed of $1200 \mathrm{rpm}$ was used to produce circular fourth-power indentations into the plates. All experimental samples in the present work were manufactured from $5 \mathrm{~mm}$ thick hot drawn mild steel sheets; 
which are more resistant to mechanical stresses incurred in the manufacturing process than cold drawn steel sheets, thus resulting in fewer internal defects. Dimensions of the produced rectangular plates were $400 \times 300 \mathrm{~mm}$, and the indentation diameter was $110 \mathrm{~mm}$ with a central hole size of $14 \mathrm{~mm}$. A piece of visco-elastic damping layer was applied to the flat surface on the back of the indentations where stated.

\subsection{Experimental set up}

Three sets of experiments were carried out in this investigation. The first tests followed the international standard ISO 3744 (see [19]) in order to determine and compare the levels of radiated sound power for the two styles of plates. These plates were then tested using a scanning laser vibrometer in order to compare the observed displacement amplitudes with the associated sound radiation. Finally, the amplitudes of response were considered at the indentation tip, again utilizing the scanning laser vibrometer.

The sound radiation experiments were conducted in the anechoic chamber of Loughborough University in order to ensure that the free field conditions were met. The plates where suspended vertically from the test rig. The tests were carried out in accordance with ISO 3744, the international engineering standard for the calculation of radiated sound power [19]. The microphone positions prescribed in ISO 3744 are shown in Fig. 2. The geometrical distances of the microphone from the sample are given in Table 1. The excitation force was applied centrally on the plate via an electromagnetic shaker with force transducer (B\&K Type 8200) attached to the plate using 'glue' and fed via a broadband signal amplifier. A microphone and a preamplifier were connected to the RT Pro Phonon analyser, and the sound pressure amplitude $p$ and the sound pressure level $L_{p}=20 \cdot \lg \left(p / p_{\text {ref }}\right)$ were obtained, 
where $p_{\text {ref }}=2 \cdot 10^{-5} \mathrm{~Pa}$ is the reference sound pressure. A schematic of the experimental set up is shown in Fig. 3.

\subsection{Calculation of the radiated sound power}

Following ISO 3744, the radiated sound power level (in $\mathrm{dB}$ ), defined as $L_{w}=$ $10 \cdot \lg \left(W / W_{\text {ref }}\right)$, was calculated according to the formula

$$
L_{w}=\bar{L}_{p f}+10 \lg \left(\frac{S}{S_{0}}\right) .
$$

In the above expressions, $W$ is the radiated sound power (in Watts), $W_{\text {ref }}=1 \cdot 10^{-12} \mathrm{~W}$ is the reference sound power, assuming that the acoustic impedance of the air is $400 \mathrm{~Pa} \cdot \mathrm{s} / \mathrm{m}, \bar{L}_{p f}$ is the measured sound pressure level (in $\mathrm{dB}$ ) averaged over the frequency band and over the microphone measurement positions on the surface of the hemisphere enclosing the radiating structure, $S$ is the area of the measurement surface (in square metres), and $S_{0}=1 \mathrm{~m}^{2}$.

The averaged sound pressure level, $\bar{L}_{p f}$, was calculated using measurements conducted in the anechoic chamber. The standard ISO 3744 requires a minimum of 20 microphone positions to obtain the spatially averaged sound pressure. Calculation of the average sound pressure level, $\bar{L}_{p f}$, over the microphone positions was performed using the expression

$$
\bar{L}_{p f}=10 \lg \left(\frac{1}{N} \sum_{i=1}^{N} 10^{0.1 L_{p i}}\right) \text {, }
$$

where $N$ is the number of microphone positions. For the case under consideration $N=20$.

Radiated sound power $W$ (in Watts) can be calculated from the sound power level $L_{w}$ (in $\mathrm{dB}$ ), by simply rearranging the definition $L_{w}=10 \cdot \mathrm{lg}\left(\mathrm{W} / \mathrm{W}_{\text {ref }}\right)$, which results in 


$$
W=W_{\text {ref }} 10^{0.1 L_{w}}
$$

The main source for error when considering results obtained using the set up above is the effect of acoustic short-circuiting due to the free edge conditions at plate edges and the absence of a baffle. It has been documented [20, 21] that these conditions can have a significant effect on the measured sound power and radiation efficiency of the plate. This effect is more pronounced at low frequencies (dipole-type radiation), and it reduces as the frequency increases. It has been also concluded that the results can be corrected using a uniform scaling factor across the frequency range. As the plates tested in this section were subjected to the same experimental conditions, the results are accurate with respect to each other. Therefore, no correction has been applied.

\subsection{Measurements of spatial distributions of vibrations over the plates}

The second set of measurements concerned spatial distributions of vibrations over the plates with and without acoustic black holes. The use of the scanning laser vibrometer Polytec OFV 056 was kindly provided by the Universite du Maine (Le Mans, France). It allowed for accurate visual representation of the mode shapes and displacement amplitudes at any given frequency in the test range. The set up for this experiment is shown in Fig. 4(a). The response was recorded by a combined accelerometer and force transducer (PCB Type 208 B02) that was attached to the opposite surface to that being scanned, using 'glue', Fig. 4(b). The laser vibrometer recorded the point accelerance of the plate along with the amplitude of deflection over the entire plate surface. A frequency range of 0-6 kHz was investigated. 
The procedure above was repeated for the final set of investigations which focused the measurements on two of the indentations. The tip deflections were then considered, concentrating on implications of the fact that one indentation had a thinner damaged tip compared to a respectively thicker undamaged tip on the other indentation.

\section{Results and discussion}

3.1 Sound radiation from a steel plate containing six circular indentations of power-law profile

This section considers sound radiation from the plate containing six indentations of power-law profile (with and without a damping layer) and from a plain reference plate. The Sound power level (in $\mathrm{dB}$ ) and Sound power (in Watts) were calculated.

As was mentioned in the Introduction, the addition of a thin visco-elastic damping layer to the indentation tip considerably increases the damping performance of the indentation of power-law profile. This is especially so when a central hole is present in the indentation [22]. It was therefore expected that the addition of such a damping layer to the indentation tip will also reduce the sound radiation from the plate.

Figure 5 shows a comparison of the Sound power level (in $\mathrm{dB}$ ) for a plate containing six indentations of power-law profile with and without a damping layer. As with the reduction in the vibration response, there is a reduction in the sound power levels of the plate when a damping layer is attached to the indentations, showing that a larger proportion of the vibration energy is not released as sound, but converted to heat via the damping layer, as 
expected. Below $1.2 \mathrm{kHz}$ the damping layer provides no increased level of sound reduction. A maximum reduction in sound power level of $8 \mathrm{~dB}$ occurs at $1.7 \mathrm{kHz}$.

The results for a plate containing six profiled circular indentations with additional damping layers, compared to a reference plate, are shown in Fig. 6 (the value of the coincidence frequency for the reference plate is $2.57 \mathrm{kHz}$ ). Below $1 \mathrm{kHz}$ there is little to no reduction in the sound pressure level, as was the case with the reduction in vibration response. Between $1-3 \mathrm{kHz}$ the sound power level response is reduced from the reference plate response by $10-18 \mathrm{~dB}$, with the maximum reduction in the sound radiated occurring at $1.6 \mathrm{kHz}$. Above $3 \mathrm{kHz}$ almost all peak responses in sound radiation are flattened.

Figure 7 shows a comparison of the radiated sound power in Watts for a plate containing six indentations of power-law profile, with and without a damping layer, compared to a plain reference plate. After $1.2 \mathrm{kHz}$ the radiated sound power in Watts for the six indentation plate with damping layers has been reduced to a level where almost all peaks seen in the reference plate have been removed. The effect of the addition of a damping layer can be seen, but it is less obvious than that seen in the sound power level plots. A maximum reduction of $5.1 \times 10^{-8}$ Watts is seen at $1.4 \mathrm{kHz}$.

From the above results, it can be concluded that making multiple indentations of powerlaw profile (with a damping layer) in a steel plate is an effective method of reducing sound radiation of a plate in the medium frequency range $(\sim 1-5 \mathrm{kHz})$. It is well known that at higher frequencies, corresponding to higher order plate modes, sound is generated less efficiently anyway due to the destructive interference [1-4]. Thus, this method, although efficient at damping higher frequency vibrations, is not expected to further reduce higher frequency sound radiation because the latter is already low due to the destructive interference caused by 
elementary sources associated with plate vibrations at higher order modes, regardless of their amplitudes. This is clearly seen in Figs. 6 and 7 above 5 kHz.

\subsection{Comparison of the sound power responses to the vibrational responses of the plates}

The amplitudes of flexural vibrations of a plate are directly linked to the amplitudes of the sound radiated by the same plate. This section considers the amplitudes of the plates response in comparison to the amplitudes of the sound radiation. A frequency range of $0-5 \mathrm{kHz}$ was used for this investigation. There main reason for this choice is that the difference in responses in sound power is little to non above the upper frequency range value. This is due to the above-mentioned well-known fact that plates as a rule radiate inefficiently at higher order vibration modes, i.e. at higher frequencies. Three resonances were selected for comparison from this frequency range; a low frequency resonance, where there is little to no difference in the sound power of the two samples, a resonance around the centre of the chosen frequency range, with a substantial difference in responses, and a resonance towards the upper limit of the frequency range. The two samples considered were the reference plate and the plate containing six indentations of power-law profile with attached damping layers.

The first resonance considered was that at $900 \mathrm{~Hz}$. Although there is a peak shift in the response, there is a minimal difference between the amplitude of the resonance in sound power and accelerance. Figure 8 shows the results for the sound power in Watts for the reference plate compared to the plate containing six indentations with damping layers and the modal response of the reference plate and of the plate containing six indentations with damping layers. A defined modal shape can be seen on both plates with little to no difference in the amplitude of the response, which is due to the fact that acoustic black holes are not 
efficient at such a low frequency. This corresponds to the limited reduction in measured sound power, as can be seen in Fig. 8(a). The effect of the indentations at this frequency serves only to slightly alter the observed mode shape.

The second resonance considered was that seen at $2.2 \mathrm{kHz}$, where a reduction in sound power of $3 \times 10^{-8}$ Watts and a reduction in accelerance of $9 \mathrm{~dB}$ from the reference plate can be seen. Figure 9 shows the results for the sound power in Watts for the reference plate at this frequency compared to the plate containing six indentations with damping layers, and the modal response of the reference plate and the plate containing six indentations with damping layers. At this frequency, the modal shape of the reference plate seen in Fig. 9(b) has been almost 'eliminated' by the plate containing six indentations of power-law profile with damping layers, Fig. 9(c). This corresponds well with the observed reduction in sound radiation seen in Fig. 9(a). Some sound radiation from the panel is still present, which can be explained by the fact that, as can be seen in Fig. 9(c), there are still non-zero displacements over the constant thickness area of the plate. These displacements increase in the indentations, and then a large amplitude increase is seen at the final $2 \mathrm{~cm}$ at the tips of the indentations. Despite these tip deflections being almost equal in amplitude to that seen on the reference plate, the average reduction of displacements over the plate with six circular indentations is great enough to result in a considerable reduction in sound radiation when compared to the reference plate.

The final resonance considered was that seen at $4.75 \mathrm{kHz}$. Figure 10 shows the results for the sound power in Watts for a reference plate compared to the plate containing six indentations with damping layers, and the modal response of the reference plate and the plate containing six indentations with damping layers. It can clearly be seen, Fig. 10(c), that other than within a radius of $3 \mathrm{~cm}$ at the centre of the indentations the amplitude of the response 
over the entire plate is zero. The mode shape seen in Fig. 10(b) had been totally 'eliminated' in Fig. 10(c). The 'active' area on the indentation plate, where a response is seen, corresponds to the area over which the damping layer was determined to be effective. The amplitude of the response in the 'active' area is approximately $1 \mathrm{~m} / \mathrm{s}^{2}$ greater than that seen on the reference plate. However, this does not affect the sound radiation of the plate as seen in Fig. 10(a). The trends described above are the same for all resonances observed during testing.

\subsection{Deflections of the tips of circular indentations of power-law profile}

It became obvious when considering the Figs. 9(c) and 10(c) and pictures for the other modes (not shown) that one of the indentations was consistently underperforming, with less than half the displacement amplitude seen in the other indentations of power-law profile. This prompted a closer physical examination of the indentation tips, where it was found that one of the indentations was slightly thicker $(0.14 \mathrm{~mm})$ and had no damage (tearing or blistering) (Fig. 11(d)), whereas the others where all similar (Fig. 11(c)) with thinner (0.10 mm) slightly damaged tips.

Earlier, when considering a wedge of power-law profile, it was found [23] that a thinner wedge tip, even if damaged, was more effective at damping flexural vibrations than a thicker undamaged tip. This conclusion was derived from the testing of a large number of wedges and analysing the response from each wedge. In the present case, with the aid of the scanning laser vibrometer, the difference in the amplitudes of the displacements of the indentation tips for these two cases of 2D acoustic black holes can be clearly seen, Fig. 11. As with a wedge of power-law profile, a circular indentation of power-law profile performs more effectively as an acoustic black hole with a thinner damaged indentation tip when compared to a slightly 
thicker undamaged indentation tip. This result confirms the strong influence of thickness of the indentation tips on the performance of the $2 \mathrm{D}$ acoustic black holes and on the resulting vibration damping and structure-borne noise.

\section{Conclusions}

A plate containing an array of six two-dimensional acoustic black holes (indentations of power-law profile covered by small pieces of damping layer) has shown a significant reduction in the level of structure-borne sound. In the medium frequency range, $1-3 \mathrm{kHz}$, the radiated sound power level was reduced from the reference plate response by $10-18 \mathrm{~dB}$.

As the frequency increases the amplitudes of deflection over the constant thickness section of a plate containing circular indentations of power-law profile tend to zero. At lower frequencies, where no reduction in sound radiation or vibration response is seen, the plate behaves as a constant thickness plate, with a little difference from the reference plate without indentations.

In the frequency range where reductions in vibration response and sound radiation are seen the plate vibration pattern changes substantially, with a noticeable amplitude reduction outside the indentations. In the higher frequency range the only displacement on the plate is seen in the last $2 \mathrm{~cm}$ towards the centres of the indentations. This corresponds to the area of maximum effectiveness of the damping layer.

Despite the indentation tip deflections almost being equal in amplitude to those seen on the reference plate, the average reduction over the plate with six circular indentations is great 
enough to result in a considerable reduction in sound radiation when compared to the reference plate.

As with a wedge of power-law profile, a circular indentation of power-law profile performs more effectively as an acoustic black hole with a thinner damaged indentation tip, when compared to an indentation with a slightly thicker and undamaged indentation tip.

\section{Acknowledgements}

The research reported here has been partly supported by the EPSRC grant EP/F009232/1. The authors are grateful to Francois Gautier of the Universite du Maine (Le Mans, France) for providing the opportunity to use their scanning laser vibrometer for some vibration measurements reported in this paper. Thanks also to Mr M. Secail-Geraud for his assistance with the laser vibrometer in Le Mans.

\section{References}

[1] Junger MC, Feit D. Sound, structures and their interaction. Cambridge MA: MIT Press; 1972.

[2] Pierce AD. Acoustics: an introduction to its physical principles and applications. New York: McGraw-Hill; 1981.

[3] Fahy FJ. Sound and structural vibration. London: Academic Press; 1985. 
[4] Kinsler LE, Frey AR, Coppens AB, Sanders JV, Fundamentals of acoustics. New York: Wiley; 2000.

[5] Cremer L, Heckl M, Ungar E, Structure-borne sound: structural vibrations and sound radiation at audio frequencies. 2nd Edition. Berlin: Springer-Verlag; 1988.

[6] Mead DJ. Passive vibration control. Chichester: John Wiley and Sons; 1998.

[7] Ross D, Ungar EE, Kerwin EM Jr. Damping of plate flexural vibrations by means of viscoelastic laminae. In: Ruzicka JE, editor. Structural Damping. Oxford: Pergamon Press: 1960. p. 49-87.

[8] Vemula C, Norris AN, Cody GD. Attenuation of waves in plates and bars using a graded impedance interface at edges. J Sound Vib 1996; 196: 107-127.

[9] Krylov VV, Tilman FJBS. Acoustic black holes for flexural waves as effective vibration dampers. J Sound Vib 2004; 274: 605-619.

[10] Krylov VV. New type of vibration dampers utilising the effect of acoustic 'black holes’. Acta Acustica united with Acustica 2004; 90: 830-837.

[11] Krylov VV, Winward RETB. Experimental investigation of the acoustic black hole effect for flexural waves in tapered plates. J Sound Vib 2007; 300: 43-49.

[12] Mironov MA, Propagation of a flexural wave in a plate whose thickness decreases smoothly to zero in a finite interval. Soviet Physics - Acoustics 1988; 34: 318-319.

[13] O’Boy DJ, Krylov VV, Kralovic V. Damping of flexural vibrations in rectangular plates using the acoustic black hole effect. J Sound Vib 2010; 329: 4672-4688. 
[14] Krylov VV. Propagation of plate bending waves in the vicinity of one- and twodimensional acoustic 'black holes'. In: Proceedings of the ECCOMAS International Conference on Computational Methods in Structural Dynamics and Earthquake Engineering (COMPDYN 2007). Rethymno, Crete, Greece, 13-16 June 2007: [CD$\mathrm{ROM}]$.

[15] Gautier F, Cuenca J, Krylov VV, Simon L. Experimental investigation of the acoustic black hole effect for vibration damping in elliptical plates. (Abstract for the Conference “Acoustics 08”, Paris, France, June 2008). J Acoust Soc Am 2008; 123(5): 3318.

[16] Georgiev VB, Cuenca J, Gautier F, Simon L, Krylov VV. Damping of structural vibrations in beams and elliptical plates using the acoustic black hole effect. J Sound Vib 2011; 330: 2497-2508.

[17] O’Boy DJ, Krylov VV. Damping of flexural vibrations in circular plates with tapered central holes. J Sound Vib 2011; 330: 2220-2236.

[18] Bowyer EP, Krylov VV, Sound radiation of rectangular plates containing tapered indentations of power-law profile. Proceedings of Meetings on Acoustics (POMA) 2013; 18: pp. 030002-13.

[19] ISO 3744: 2009, Acoustics - Determination of sound power levels of noise sources using sound pressure - Engineering method in an essentially free field over a reflecting plane. Brussels: International Organization for Standardization; 2009.

[20] Putra A, Thompson DJ, Sound radiation from rectangular baffled and unbaffled plates. Applied Acoustics 2010; 71: 1113- 1125. 
[21] Oppenheimer CH, Dubowsky SA. Radiation efficiency for unbaffled plates with experimental validation. J Sound Vib 1997; 199: 473- 489.

[22] O’Boy DJ, Bowyer EP, Krylov VV. Point mobility of a cylindrical plate incorporating a tapered hole of power-law profile. J Acoust Soc Am 2011; 129: 3475-3482.

[23] Bowyer EP, O’Boy DJ, Krylov VV, Horner JL. Effect of geometrical and material imperfections on damping flexural vibrations in plates with attached wedges of power law profile. Applied Acoustics 2010; 73: 514-523. 


\section{Figure captions}

Fig. 1. (a) Plain reference plate, (b) Plate containing six indentations of powerlaw profile.

Fig. 2. Microphone positions in relation to the sample in accordance with the ISO 3744 standard.

Fig. 3. Schematic of the sound radiation set up.

Fig. 4. (a) Experimental set up, (b) Locations of the shaker (Force) and of the accelerometer (Response) on an experimental sample.

Fig. 5. Sound power level comparison for a plate containing six indentations of power-law profile with (black line) and without (grey line) a damping layer.

Fig. 6. Sound power level comparison for a plate containing six indentations of power-law profile with a damping layer (black line) compared to a reference plate (grey line). 
Fig. 7. Sound power in Watts, comparison for a plate containing six indentations of power-law profile with (black line) and without (grey line) a damping layer compared to a plain reference plate (dashed line).

Fig. 8. Results for the resonant peak at $900 \mathrm{~Hz}$ : (a) Sound power in Watts for a reference plate (dashed line) compared to the plate containing six indentations with damping layer, (b) Modal response of the reference plate, (c) Modal response of the plate containing six indentations with damping layers, (d) Amplitude of response; key.

Fig. 9. Results for the resonant peak at $2.2 \mathrm{kHz}$ : (a) Sound power in Watts for a reference plate (dashed line) compared to the plate containing six indentations with damping layers, (b) Modal response of the reference plate, (c) Modal response of the plate containing six indentations with damping layers, (d) Amplitude of response; key.

Fig. 10. Results for the resonant peak at $4.75 \mathrm{kHz}$ : (a) Sound power in Watts for a reference plate (dashed line) compared to the plate containing six indentations with damping layers, (b) Modal response of the reference plate, (c) Modal response of the plate containing six indentations with damping layers, (d) Amplitude of response; key. 
Fig. 11. (a) Displacement of the indentation shown in c, (b) Displacement of the indentation shown in d, (c) Circular indentation with a thinner damaged tip, (d) Circular indentation with a thicker undamaged tip. 


\section{Table captions}

Table 1. Geometrical distances of a microphone from the centre point of the plate (m). 


\section{Figures}

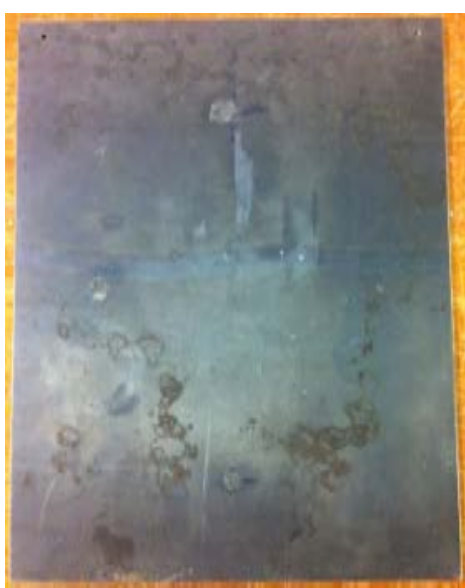

(a)

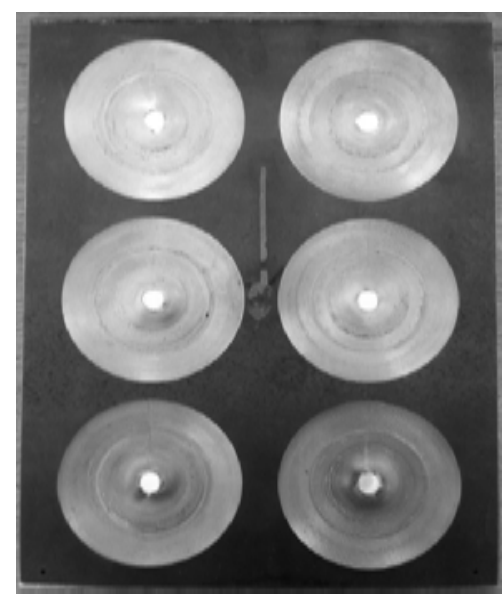

(b)

Fig. 1. (a) Plain reference plate, (b) Plate containing six indentations of power-law profile. 


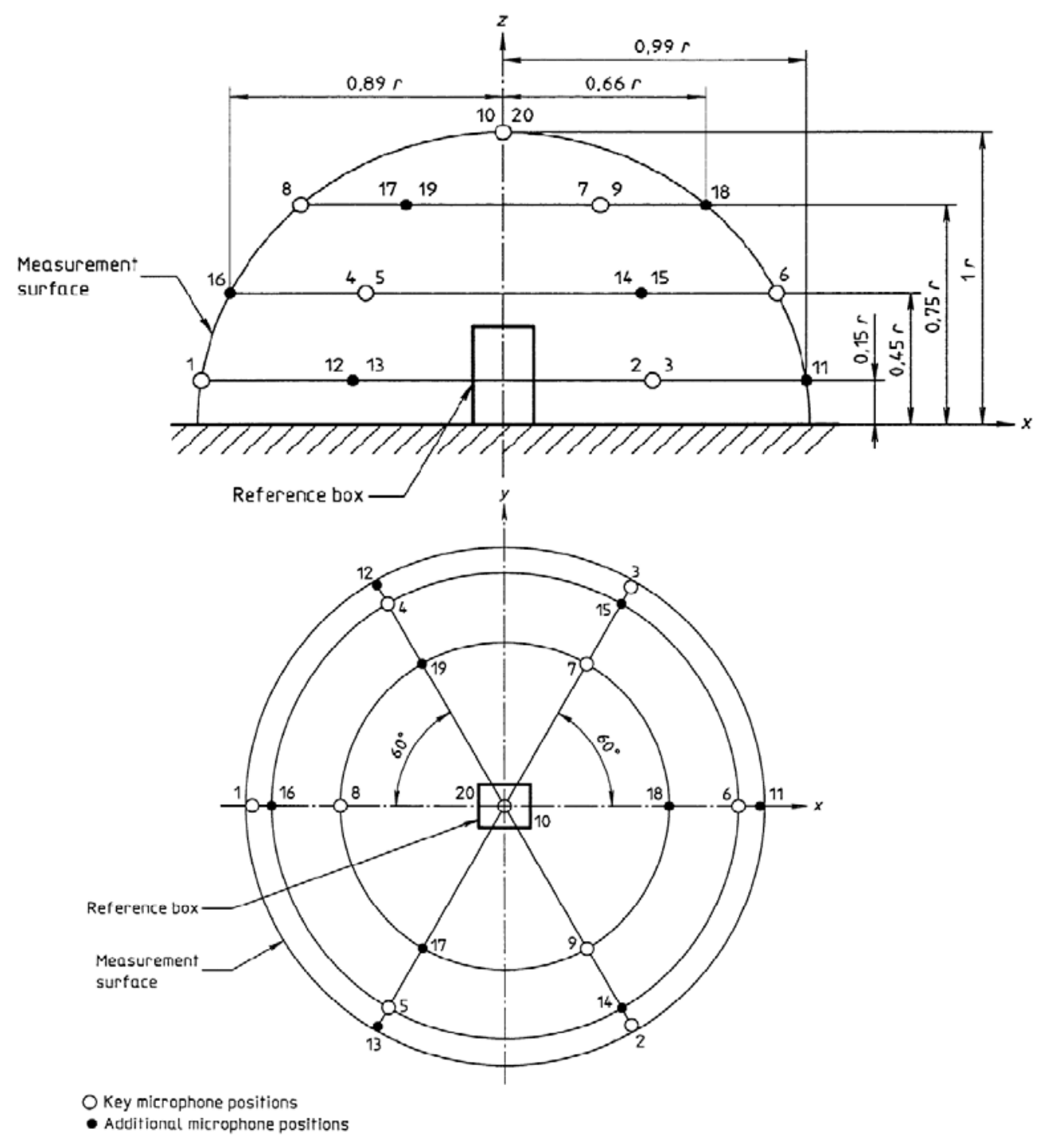

Fig. 2. Microphone positions in relation to the sample in accordance with the ISO 3744 standard. 


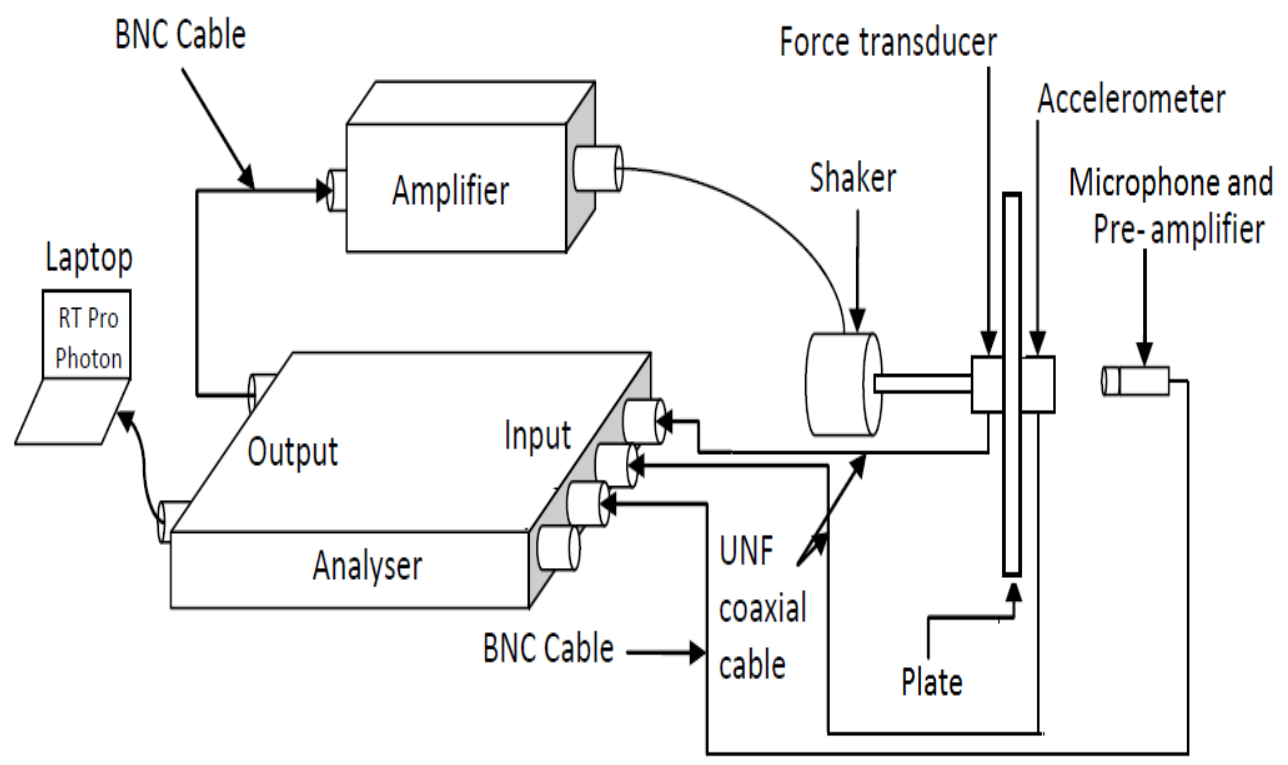

Fig. 3. Schematic of the sound radiation set up. 


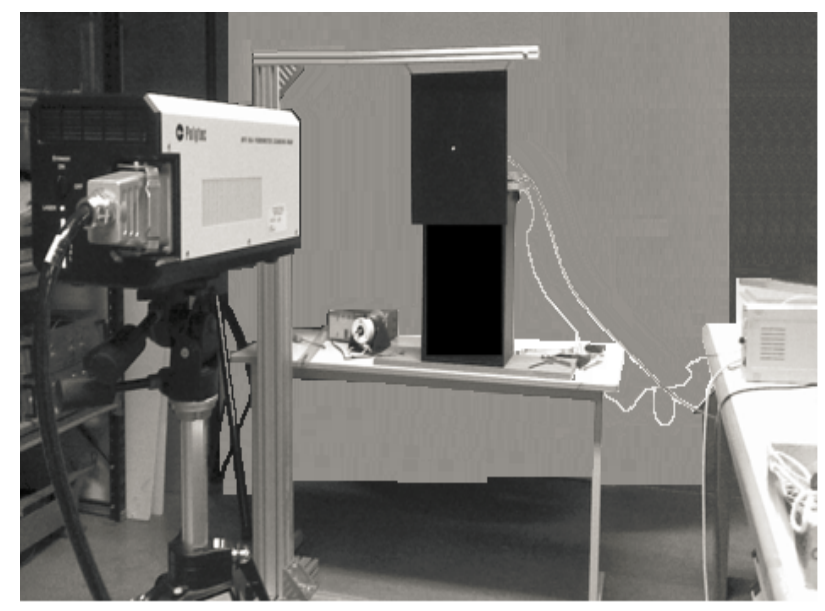

(a)

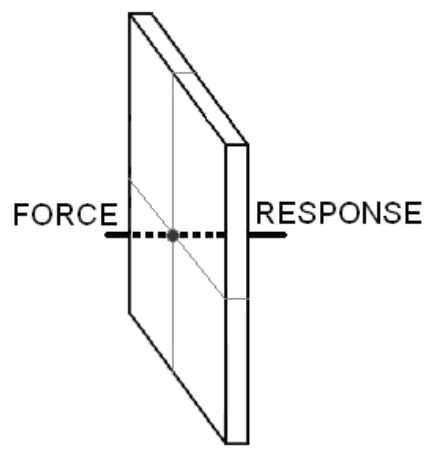

(b)

Fig. 4. Experimental set up, (b) Locations of the shaker (Force) and of the accelerometer (Response) on an experimental sample. 


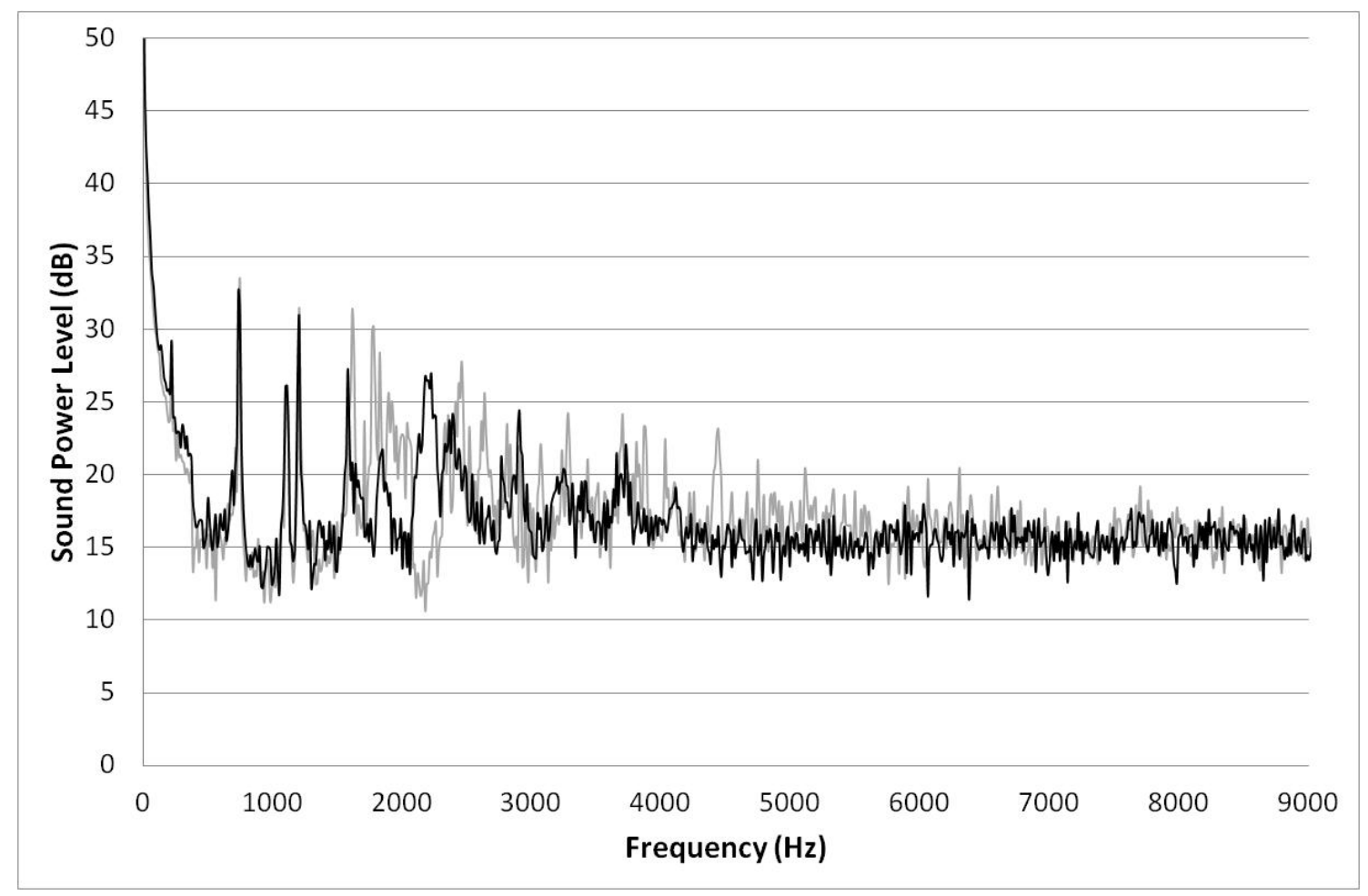

Fig. 5. Sound power level comparison for a plate containing six indentations of power-law profile with (black line) and without (grey line) a damping layer. 


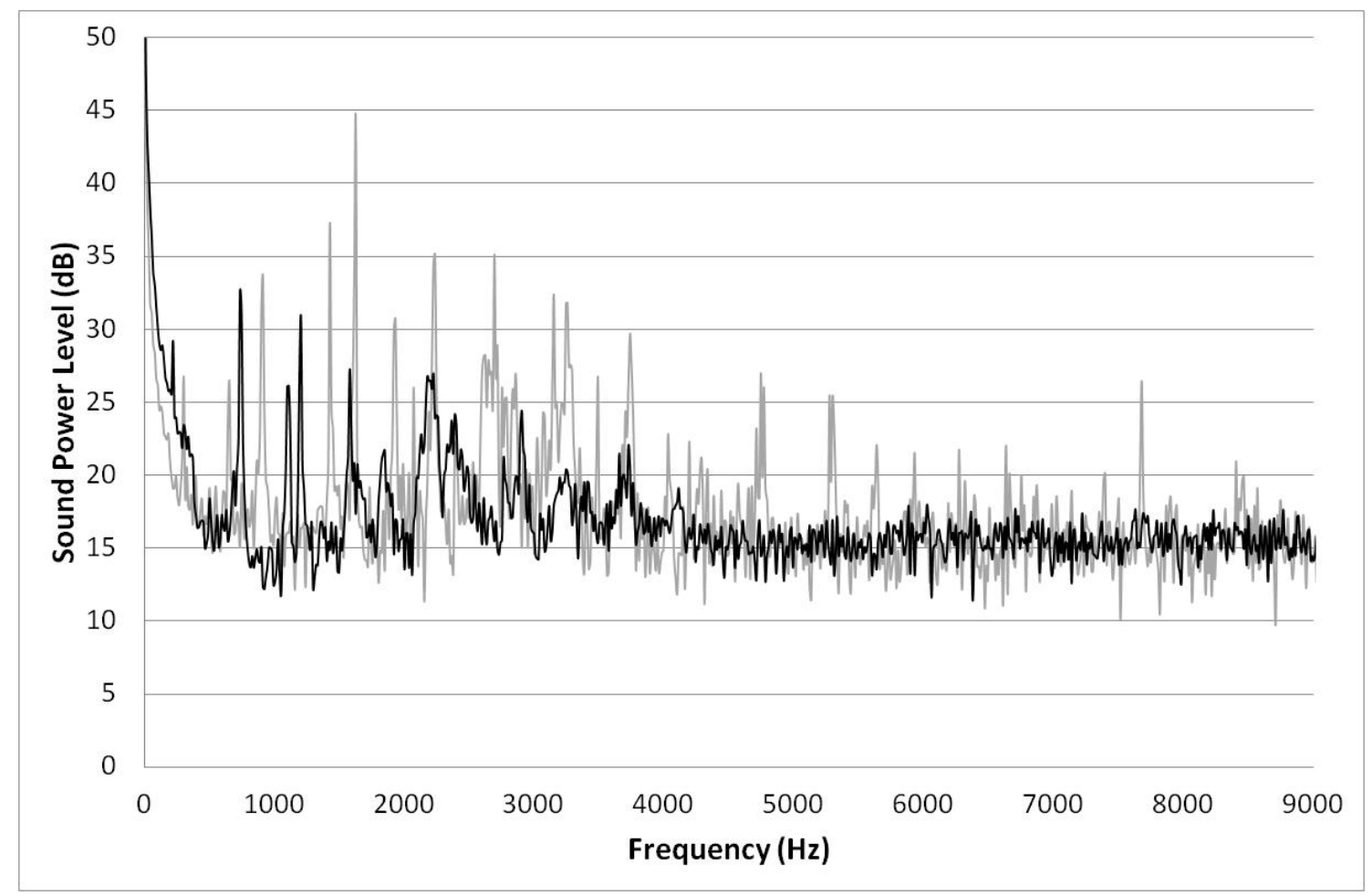

Fig. 6. Sound power level comparison for a plate containing six indentations of power-law profile with a damping layer (black line) compared to a reference plate (grey line). 


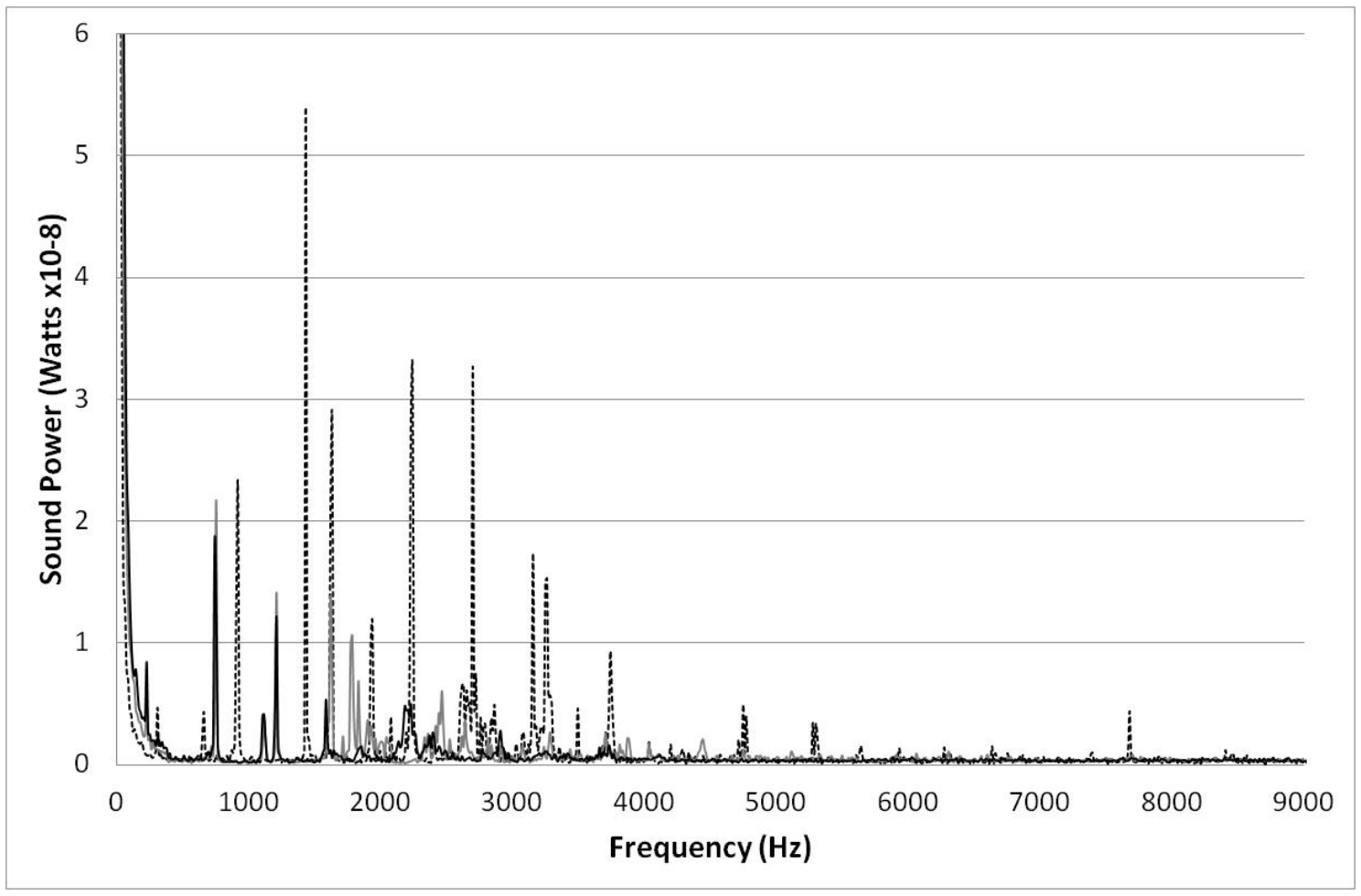

Fig. 7. Sound power in Watts, comparison for a plate containing six indentations of power-law profile with (black line) and without (grey line) a damping layer, compared to a plain reference plate (dashed line). 


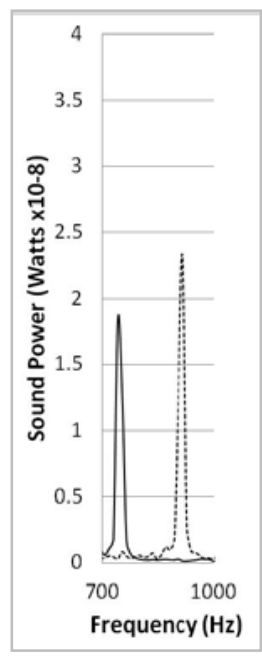

(a)

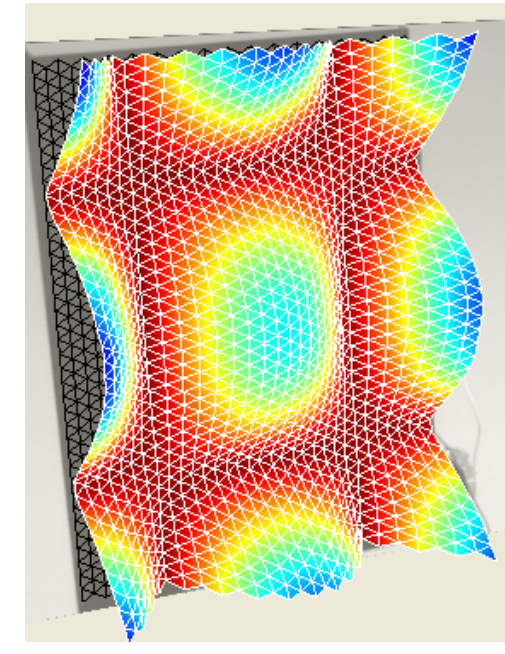

(b)

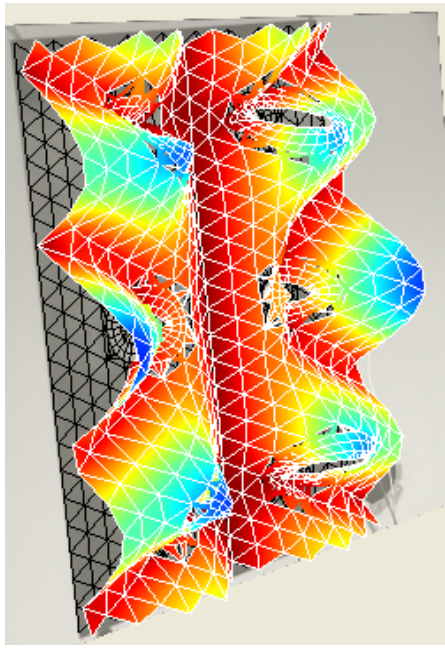

(c)

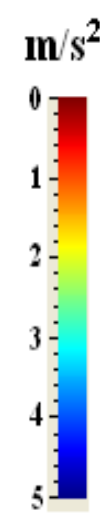

(d)

Fig. 8. Results for the resonant peak at $900 \mathrm{~Hz}$ : (a) Sound power in Watts for the reference plate (dashed line) compared to the plate containing six indentations with damping layer, (b) Modal response of the reference plate, (c) Modal response of the plate containing six indentations with damping layers, (d) Amplitude of response; key. 


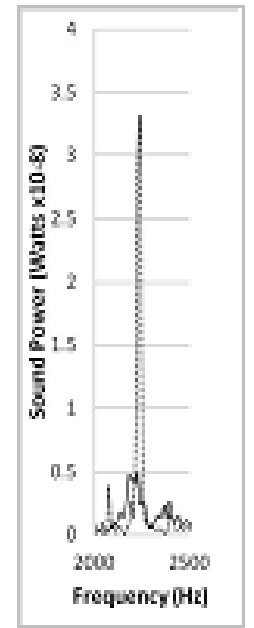

(a)

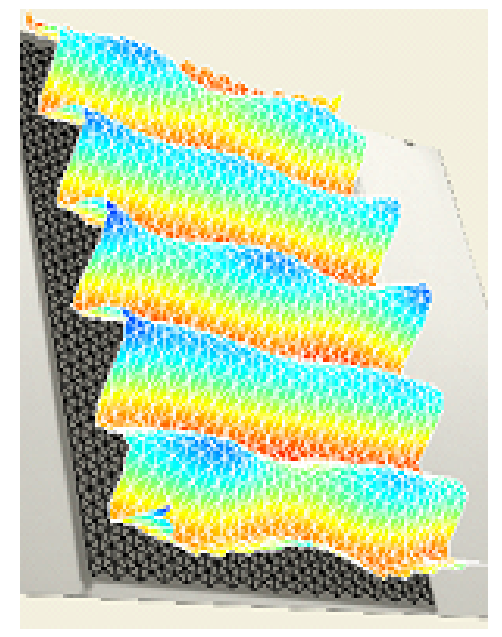

(b)

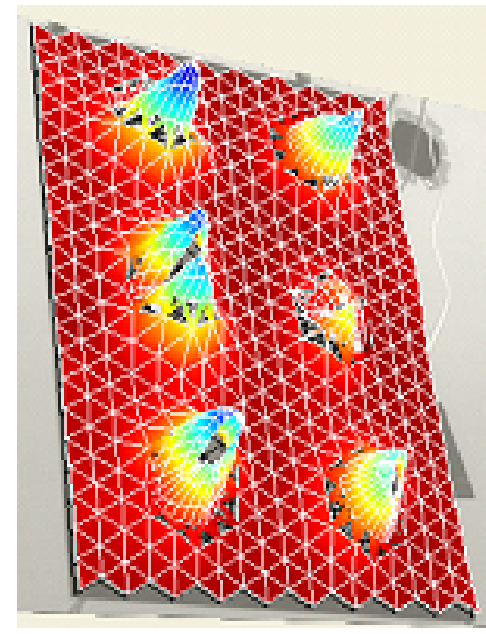

(c)

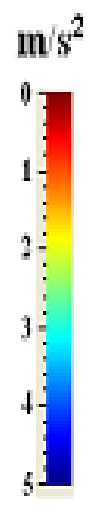

(d)

Fig. 9. Results for the resonant peak at $2.2 \mathrm{kHz}$ : (a) Sound power in Watts for the reference plate (dashed line) compared to the plate containing six indentations with damping layers, (b) Modal response of the reference plate, (c) Modal response of the plate containing six indentations with damping layers, (d) Amplitude of response; key. 


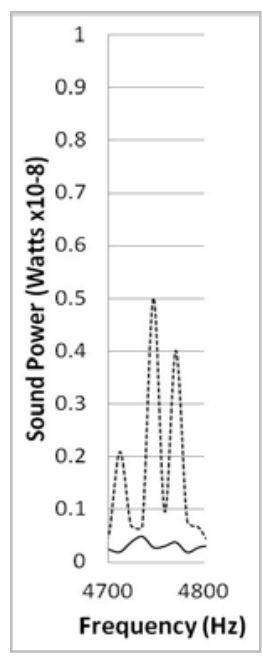

(a)

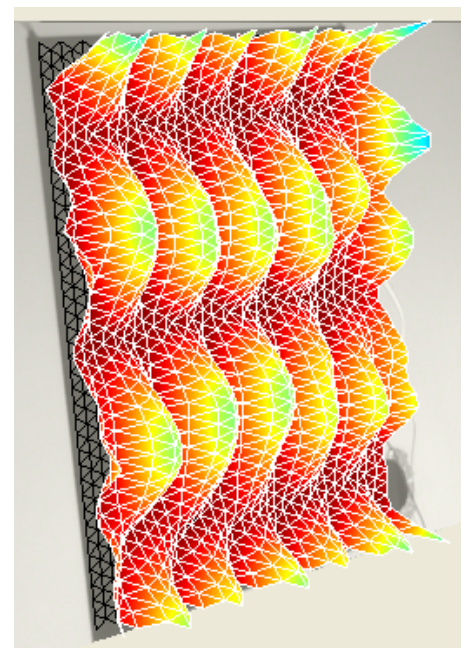

(b)

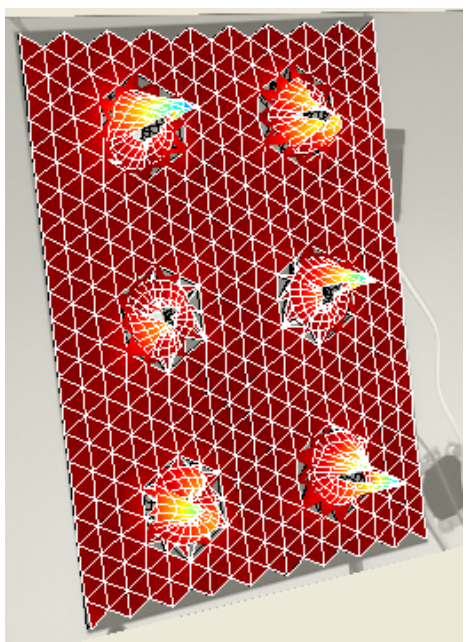

(c)

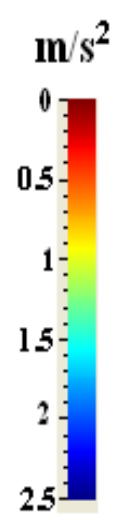

(d)

Fig. 10. Results for the resonant peak at $4.75 \mathrm{kHz}$ : (a) Sound power in Watts for the reference plate (dashed line) compared to the plate containing six indentations with damping layers, (b) Modal response of the reference plate, (c) Modal response of the plate containing six indentations with damping layers, (d) Amplitude of response; key. 


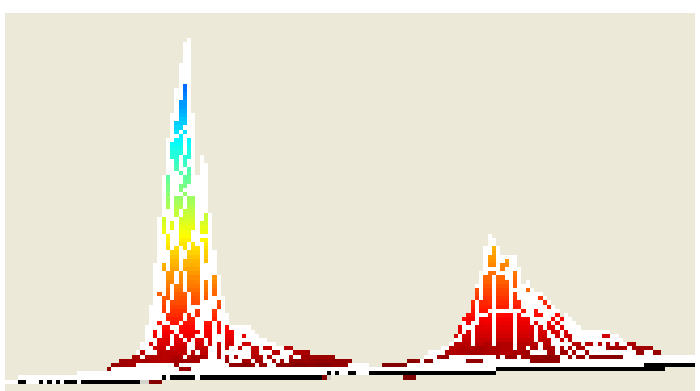

(a) (b)

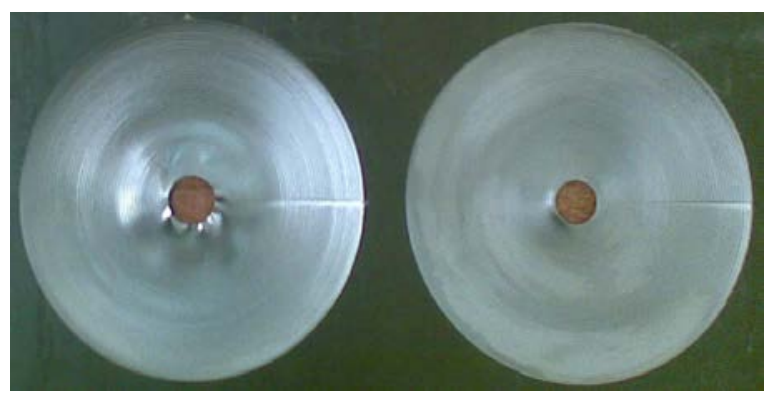

(c)

(d)

Fig. 11. (a) Displacement of the indentation shown in c, (b) Displacement of the indentation shown in d, (c) Circular indentation with a thinner damaged tip, (d) Circular indentation with a thicker undamaged tip. 


\section{Tables}

\begin{tabular}{|c|c|c|c|c|c|c|c|}
\hline Position & $\boldsymbol{X}$ & $\boldsymbol{y}$ & $\mathbf{z}$ & Position & $\boldsymbol{X}$ & $\boldsymbol{y}$ & $\mathbf{z}$ \\
\hline $\mathbf{1}$ & 1 & 0 & 0.68 & $\mathbf{1 1}$ & -1 & 0 & 0.68 \\
\hline $\mathbf{2}$ & -0.5 & 0.86 & 0.6 & $\mathbf{1 2}$ & 0.5 & -0.86 & 0.6 \\
\hline $\mathbf{3}$ & -0.5 & -0.86 & 0.6 & $\mathbf{1 3}$ & 0.5 & 0.86 & 0.66 \\
\hline $\mathbf{4}$ & 0.45 & -0.77 & 0.98 & $\mathbf{1 4}$ & -0.45 & 0.77 & 0.98 \\
\hline $\mathbf{5}$ & 0.45 & 0.77 & 0.98 & $\mathbf{1 5}$ & -0.45 & -0.77 & 0.98 \\
\hline $\mathbf{6}$ & -0.89 & 0 & 0.98 & $\mathbf{1 6}$ & 0.89 & 0 & 0.98 \\
\hline $\mathbf{7}$ & -0.33 & -0.57 & 1.28 & $\mathbf{1 7}$ & 0.33 & 0.57 & 1.28 \\
\hline $\mathbf{8}$ & 0.66 & 0 & 1.28 & $\mathbf{1 8}$ & -0.66 & 0 & 1.28 \\
\hline $\mathbf{9}$ & -0.33 & 0.57 & 1.28 & $\mathbf{1 9}$ & 0.33 & -0.57 & 1.28 \\
\hline $\mathbf{1 0}$ & 0 & 0 & 1.53 & $\mathbf{2 0}$ & 0 & 0 & 1.53 \\
\hline
\end{tabular}

Table 1. Geometrical distances of a microphone from the centre point of the plate (m). 